\title{
Comparative Numerical Study of SBA (Somé Blaise-Abbo) Method and Homotopy Perturbation Method (HPM) on Biomathematical Models Type Lotka-Volterra
}

\author{
Bakari ABBO ${ }^{1}$, BAGAYOGO Moussa $^{2}$, MINOUNGOU Youssouf $^{3}$, Youssouf PARE ${ }^{4}$ \\ ${ }^{1}$ Université de NDjaména, NDjaména, Tchad \\ ${ }^{2}$ Centre Universitaire Polytechnique de Kaya, Burkina-Faso \\ ${ }^{3}$ Institut Des Sciences, Ouagadougou, Burkina-Faso \\ ${ }^{4}$ Université Joseph Ki-Zerbo, Ouagadougou, Burkina-Faso \\ Correspondence: Youssouf PARE, Université Joseph Ki-Zerbo, Ouagadougou, Burkina-Faso.
}

Received: December 24, 2020 Accepted: March 17, 2021 Online Published: April 14, 2021

doi:10.5539/jmr.v13n3p22

URL: https://doi.org/10.5539/jmr.v13n3p22

\section{Abstract}

In this work the Homotopy Perturbation Method (HPM) is used to find an exact or approximate solutions of Lotka-Volterra models. Then we compare the HPM solution with the solution given by SBA (Somé Blaise Abbo) method.

Keywords: Lotka-Volterra models, Homotopy Perturbation Method (HPM), SBA (Somé Blaise Abbo) method

\section{Introduction}

We are interested in the resolution of nonlinear systems of general form :

$$
\left\{\begin{array}{l}
\frac{d u}{d t}=f(u, v)+g(u, v) \\
\frac{d v}{d t}=\varphi(u, v)+\psi(u, v)
\end{array}\right.
$$

in a suitable Hilbert space, where $f(u, v)$ et $\varphi(u, v)$ are the linear functions and $\varphi(u, v)$ and $\psi(u, v)$ are nonlinear fonction. These systems model a large number of phenomena in biomathematics. They are also Lotka-Volterra equations type of the form:

$$
\left\{\begin{array}{l}
\frac{d u}{d t}=a u+b u v \\
\frac{d v}{d t}=-c v+b u v
\end{array}\right.
$$

In this article, we use Homotopy Perturbation Method (BAGAYOGO, M, 2019; M.S.H. Chowdhury \& et al., 2010) to solve Lotka-Volterra models. Then we compare the HPM solutions with the solution given by SBA (Som Blaise Abbo) method (MINOUNGOU,Y., 2019; PARE, Y., 2010). SBA (MINOUNGOU,Y., 2019; PARE, Y., 2010; YARO, R., 2016) method is a numerical method for obtaining exact or approximate solutions of linear or nonlinear equations.

The paper is organized as follows: in the following section the homotopy perturbation method is explained. In Section 3 we solve two problems. Numerical results are reported in Section 4. Finally, the paper is concluded in Section 5.

\section{HPM for System of ODEs}

To illustrate the basic idea of the HPM for system of PDEs, we consider the following non-homogeneous, non- linear system of ODEs ( M.S.H. Chowdhury \& et al., 2010; M.S.H. Chowdhury \& et al., 2015).

$$
\begin{aligned}
& \frac{d u_{1}}{d t}+g_{1}\left(t, u_{1}, u_{2}, \cdots, u_{m}\right)=f_{1}(t) \\
& \frac{d u_{2}}{d t}+g_{2}\left(t, u_{1}, u_{2}, \cdots, u_{m}\right)=f_{2}(t)
\end{aligned}
$$




$$
\frac{d u_{m}}{d t}+g_{m}\left(t, u_{1}, u_{2}, \cdots, u_{m}\right)=f_{m}(t)
$$

subject to the initial conditions

$$
u_{1}(0)=c_{1} u_{2}(0)=c_{2}, \cdots, u_{m}(0)=c_{m}
$$

where $u_{m}=u_{m}(t)$ and $f_{m}=f_{m}(t)$

First write system (1)-(3) in the operator form:

$$
\begin{aligned}
L\left(u_{1}\right)+N_{1}\left(u_{1}, u_{2}, \cdots, u_{m}\right)-f_{1} & =0 \\
L\left(u_{2}\right)+N_{2}\left(u_{1}, u_{2}, \cdots, u_{m}\right)-f_{2} & =0 \\
& \vdots \\
L\left(u_{m}\right)+N_{m}\left(u_{1}, u_{2}, \cdots, u_{m}\right)-f_{m} & =0
\end{aligned}
$$

subject to the initial conditions (4), where $L(\cdot)=\frac{d(\cdot)}{d t}$ is linear operator and $N_{1}, N_{2}, \cdots, N_{m}$ are nonlinear operators.

According to HPM (BAGAYOGO, M., 2019; BAGAYOGO, M., 2018; BAGAYOGO, M. \& et al., 2019), we construct a homotopy for (5)-(7) which satisfies the following relations:

$$
\begin{aligned}
L\left(u_{1}\right)-L\left(v_{1}\right)+p L\left(v_{1}\right)+p\left[N_{1}\left(u_{1}, u_{2}, \cdots, u_{m}\right)-f_{1}\right] & =0 \\
L\left(u_{2}\right)-L\left(v_{2}\right)+p L\left(v_{2}\right)+p\left[N_{2}\left(u_{1}, u_{2}, \cdots, u_{m}\right)-f_{2}\right] & =0 \\
& \vdots \\
L\left(u_{m}\right)-L\left(v_{m}\right)+p L\left(v_{m}\right)+p\left[N_{m}\left(u_{1}, u_{2}, \cdots, u_{m}\right)-f_{m}\right] & =0
\end{aligned}
$$

where $p \in[0,1]$ is an embedding parameter and $v_{1}, v_{2}, \cdots, v_{m}$ are initial approximations which satisfying the given conditions. Consider the initial approximations as follows:

$$
\begin{aligned}
u_{1,0}(t) & =v_{1}(t)=u_{1}(0)=c_{1} \\
u_{2,0}(t) & =v_{2}(t)=u_{2}(0)=c_{2} \\
& \vdots \\
u_{m, 0}(t) & =v_{m}(t)=u_{m}(0)=c_{m}
\end{aligned}
$$

and

$$
\begin{aligned}
u_{1}(t) & =u_{1,0}(t)+p u_{1,1}(t)+p^{2} u_{1,2}(t)+\cdots \\
u_{2}(t) & =u_{2,0}(t)+p u_{2,1}(t)+p^{2} u_{2,2}(t)+\cdots \\
& \vdots \\
u_{m}(t) & =u_{m, 0}(t)+p u_{m, 1}(t)+p^{2} u_{m, 2}(t)+\cdots
\end{aligned}
$$

where $u_{i, j}(i=1,2, \cdots, m ; j=1,2 ;, \cdots, \cdots)$ are functions yet to be determined. Substituting (9) into (8) arranging the coeffcients of the same powers of $p$, obtain

$$
\begin{aligned}
L\left(u_{1,1}\right)+L\left(v_{1}\right)+N_{1}\left(u_{1,0}, u_{2,0}, \cdots, u_{m, 0}\right)-f_{1} & =0, u_{1,1}(0)=0 \\
L\left(u_{2,1}\right)+L\left(v_{2}\right)+N_{2}\left(u_{1,0}, u_{2,0}, \cdots, u_{m, 0}\right)-f_{2} & =0, u_{2,1}(0)=0 \\
& \vdots \\
L\left(u_{m, 1}\right)+L\left(v_{m}\right)+N_{m}\left(u_{1,0}, u_{2,0}, \cdots, u_{m, 0}\right)-f_{m} & =0, u_{m, 1}(0)=0
\end{aligned}
$$$$
L\left(u_{1,2}\right)+N_{1}\left(u_{1,1}, u_{2,1}, \cdots, u_{m, 1}\right)-f_{1}=0, u_{1,2}(0)=0
$$ 


$$
\begin{aligned}
L\left(u_{2,2}\right)+N_{2}\left(u_{1,1}, u_{2,1}, \cdots, u_{m, 1}\right)-f_{2} & =0, u_{2,2}(0)=0 \\
& \vdots \\
L\left(u_{m, 2}\right)+N_{m}\left(u_{1,1}, u_{2,1}, \cdots, u_{m, 1}\right)-f_{m} & =0, \quad u_{m, 2}(0)=0
\end{aligned}
$$

etc

Now solve the above systems of equations for the unknowns $u_{i, j}(i=1,2, \cdots, m ; j=1,2, \cdots, \cdots)$. Therefore, according to HPM the $n$-term approximations for the solutions of (5)-(7) can be expressed as

$$
\begin{aligned}
\phi_{1, n}(t) & =u_{1}(t)=\lim _{p \rightarrow 1} u_{1}(t)=\sum_{k=0}^{n-1} u_{1, k}(t) \\
\phi_{2, n}(t) & =u_{2}(t)=\lim _{p \rightarrow 1} u_{2}(t)=\sum_{k=0}^{n-1} u_{2, k}(t) \\
& \vdots \\
\phi_{m, n}(t) & =u_{m}(t)=\lim _{p \rightarrow 1} u_{m}(t)=\sum_{k=0}^{n-1} u_{m, k}(t)
\end{aligned}
$$

\section{Application}

\subsection{Example 1}

Consider a Lotka-Volterra model which describe predators-prey interactions (ABBO, B., 2007) :

$$
\left\{\begin{array}{l}
\frac{d u}{d t}=\alpha u-c_{2} u^{2}+c_{1} u v \\
\frac{d v}{d t}=\alpha v-c_{1} v^{2}-c_{2} u v
\end{array}\right.
$$

with the initial conditions $u(0)=c_{1}$ et $v(0)=c_{2}$.

The exact solution of (10) obtained by SBA method is (ABBO, B., 2007) :

$$
(u, v)=\left(c_{1} e^{\alpha t}, c_{2} e^{\alpha t}\right)
$$

In order to apply the homotopy perturbation method, we construct the following homotopy equations :

$$
\begin{aligned}
& (1-p)\left[\frac{d u}{d t}-\alpha u\right]+p\left[\frac{d u}{d t}-\alpha u+c_{2} u^{2}+c_{1} u v\right]=0 \\
& (1-p)\left[\frac{d v}{d t}-\alpha v\right]+p\left[\frac{d v}{d t}-\alpha v+c_{1} v^{2}+c_{2} u v\right]=0
\end{aligned}
$$

We obtain the following homotopy system:

$$
\left\{\begin{array}{c}
\frac{d u}{d t}-\alpha u+p c_{2} u^{2} u-p c_{1} u v=0 \\
\frac{d v}{d t}-\alpha v-c_{1} v^{2}+c_{2} p u v=0
\end{array}\right.
$$

Assume the solution of (10) to be in the form :

$$
\begin{aligned}
& u(t)=u_{0}+p u_{1}+p^{2} u_{2}+p^{3} u_{3}+\cdots \\
& v(t)=v_{0}+p v_{1}+p^{2} v_{2}+p^{3} v_{3}+\cdots
\end{aligned}
$$


Substituting (12) into (11) and equating the coefficients of like $p$, we get the following set of differential equations :

$$
\begin{aligned}
& p^{0}:\left\{\begin{array}{l}
\frac{d u_{0}}{d t}-\alpha u_{0}=0 \\
\frac{d v_{0}}{d t}-\alpha v_{0}=0
\end{array} \quad u_{0}(0)=c_{1}, \quad v_{0}(0)=c_{2}\right. \\
& p^{1}:\left\{\begin{array}{l}
\frac{d u_{1}}{d t}-\alpha u_{1}-c_{1} u_{0} v_{0}+c_{2} u_{0}^{2}=0 \\
\frac{d v_{1}}{d t}-\alpha v_{1}-c_{1} v_{0}^{2}+c_{2} u_{0} v_{0}=0
\end{array}\right. \\
& p^{2}: \begin{cases}\frac{d u_{2}}{d t}-\alpha u_{2}-c_{1} u_{0} v_{1}-c_{1} u_{1} v_{0}+2 c_{2} u_{0} u_{1}=0 \\
\frac{d v_{2}}{d t}-\alpha v_{2}-2 c_{1} v_{0} v_{1}+c_{2} u_{0} v_{1}+c_{2} u_{1} v_{0}=0\end{cases}
\end{aligned}
$$

Solving the above equations, we obtain

$$
\begin{aligned}
& u_{0}(t)=c_{1} e^{\alpha t} \\
& v_{0}(t)=c_{2} e^{\alpha t} \\
& u_{1}(t)=0 \\
& v_{1}(t)=0
\end{aligned}
$$

For $n \geq 1$, we have $u_{n}(t)=v_{n}(t)=0$

Hence the solution of (10) by HPM is given by

$$
\begin{aligned}
u(t) & =\lim _{p \rightarrow 1}\left[u_{0}(t)+p u_{1}(t)+p^{2} u_{2}(t)+\cdots\right] \\
& =\lim _{p \rightarrow 1} u_{0}(t) \\
& =u_{0}(t) \\
v(t) & \simeq \lim _{p \rightarrow 1}\left[v_{0}(t)+p v_{1}(t)+p^{2} v_{2}(t)+\cdots\right] \\
& =\lim _{p \rightarrow 1} v_{0}(t) \\
& =v_{0}(t)
\end{aligned}
$$

Therefore

$$
u(t)=c_{1} e^{\alpha t} \quad \text { et } \quad v(t)=c_{2} e^{\alpha t}
$$

\subsection{Example 2}

Consider a LotKa-Volterra model, describing competitors (YARO, R., 2016)

$$
\left\{\begin{array}{l}
\frac{d u}{d t}=\frac{1}{7}(3 u+10 v)+2 u v\left(u+v-(\alpha+\beta) e^{t}\right) \\
\frac{d v}{d t}=\frac{1}{7}(4 u-3 v)-2 u v\left(2 u-5 v-(\alpha+\beta) e^{-t}\right)
\end{array} \quad t \geq 0\right.
$$

with the initial conditions $u(0)=\alpha=6 \cdot 10^{6}$ and $v(0)=\beta=10^{6}$ where $\alpha=6 \beta$ 
The exact solution of (16) obtained by SBA method is (YARO, R., (2016) :

$$
(u, v)=\left(\alpha \operatorname{ch}(t)+\frac{1}{7}(3 \alpha+10 \beta) \operatorname{sh}(t), \beta \operatorname{ch}(t)+\frac{1}{7}(4 \alpha-3 \beta) \operatorname{sh}(t)\right)
$$

According to HPM, we construct the following homotopy equations :

$$
\begin{aligned}
& (1-p)\left[\frac{d u}{d t}-\frac{3}{7} u\right]+p\left[\frac{d u}{d t}-\frac{3}{7} u-\frac{1}{7}(3 u+10 v)-2 u v\left(u+v-(\alpha+\beta) e^{t}\right)\right]=0 \\
& (1-p)\left[\frac{d v}{d t}-\frac{4}{7} v\right]+p\left[\frac{d v}{d t}-\frac{4}{7} v-\frac{1}{7}(4 u-3 v)+2 u v\left(2 u-5 v-(\alpha+\beta) e^{-t}\right)\right]=0
\end{aligned}
$$

We obtain the homotopy system:

$$
\left\{\begin{array}{c}
\frac{d u}{d t}-\frac{3}{7} u-p \frac{10}{7} v-2 p u v\left(u+v-(\alpha+\beta) e^{t}\right)=0 \\
\frac{d v}{d t}-\frac{4}{7} v-p \frac{4}{7} u+2 p u v\left(2 u-5 v-(\alpha+\beta) e^{-t}\right)=0
\end{array}\right.
$$

Suppose the solution of (16) have the form:

$$
\begin{aligned}
& u(t)=u_{0}+p u_{1}+p^{2} u_{2}+p^{3} u_{3}+\cdots \\
& v(t)=v_{0}+p v_{1}+p^{2} v_{2}+p^{3} v_{3}+\cdots
\end{aligned}
$$

Then substituting (18) into (17) and rearanging based on powers of $p$-terms, we have:

$$
\begin{aligned}
& p^{0}:\left\{\begin{array}{l}
\frac{d u_{0}}{d t}-\frac{3}{7} u_{0}=0 \\
\frac{d v_{0}}{d t}-\frac{4}{7} v_{0}=0
\end{array} \quad u_{0}(0)=\alpha, \quad v_{0}(0)=\beta\right. \\
& p^{1}:\left\{\begin{array}{l}
\frac{d u_{1}}{d t}-\frac{3}{7} u_{1}+2 u_{0} v_{0}\left[u_{0}+v_{0}+(\alpha+\beta) e^{t}\right]-\frac{10}{7} v_{0}=0 \\
\frac{d v_{1}}{d t}-\frac{4}{7} v_{1}+2 u_{0} v_{0}\left[2 u_{0}-5 v_{0}-(\alpha+\beta) e^{-t}\right]+\frac{4}{7} u_{0}=0
\end{array}\right. \\
& p^{2}:\left\{\begin{array}{c}
\frac{d u_{2}}{d t}-\frac{3}{7} u_{2}+2 u_{0} v_{1}(\alpha+\beta) e^{t}+2 u_{1} v_{0}(\alpha+\beta) e^{t}-4 u_{0} v_{0}\left(u_{1}+v_{1}\right) \\
-2 u_{0} v_{1}\left(u_{0}+v_{0}\right)-\frac{10}{7} v_{1}=0 \\
\begin{array}{c}
\frac{d v_{2}}{d t}-\frac{4}{7} v_{2}-2 u_{0} v_{1}(\alpha+\beta) e^{-t}+2 u_{1} v_{0}(\alpha+\beta) e^{-t}-4 u_{0} v_{0}\left(2 u_{1}-5 v_{1}\right) \\
+2 u_{0} v_{1}\left(2 u_{0}-5 v_{0}\right)+\frac{4}{7} u_{1}=0
\end{array} \quad 0
\end{array}\right.
\end{aligned}
$$

The solutions of above equations are as follows:

$$
\begin{aligned}
u_{0}(t)= & \alpha e^{\frac{3 t}{7}} \\
v_{0}(t)= & \beta e^{\frac{4 t}{7}} \\
u_{1}(t)= & -\frac{14 e^{2 t} \alpha \beta^{2}}{11}+\frac{7 e^{\frac{11 t}{7}} \alpha \beta^{2}}{4}-\frac{21 e^{\frac{3 t}{7}} \alpha \beta^{2}}{44}-\frac{14 e^{2 t} \alpha^{2} \beta}{11}+2 e^{\frac{10 t}{7}} \alpha^{2} \beta \\
& -\frac{8 e^{\frac{3 t}{7}} \alpha^{2} \beta}{11}+10 e^{\frac{4 t}{7}} \beta-10 e^{\frac{3 t}{7}} \beta
\end{aligned}
$$




$$
\begin{aligned}
& v_{1}(t)=10 e^{\frac{11 t}{7}} \alpha \beta^{2}-\frac{13 e^{\frac{4 t}{7}} \alpha \beta^{2}}{2}-\frac{7 \alpha \beta^{2}}{2}-\frac{14 e^{\frac{10 t}{7}} \alpha^{2} \beta}{3}+\frac{49 e^{\frac{4 t}{7}} \alpha^{2} \beta}{6}-\frac{7 \alpha^{2} \beta}{2} \\
& -4 e^{\frac{4 t}{7}} \alpha+4 e^{\frac{3 t}{7}} \alpha \\
& u_{2}(t)=\frac{28 e^{\frac{25 t}{7}} \alpha \beta^{4}}{33}-\frac{931 e^{\frac{22 t}{7}} \alpha \beta^{4}}{396}+\frac{49 e^{\frac{19 t}{7}} \alpha \beta^{4}}{30}+\frac{147 e^{2 t} \alpha \beta^{4}}{220}-\frac{21 e^{\frac{11 t}{7}} \alpha \beta^{4}}{22} \\
& +\frac{7 e^{\frac{4 t}{7}} \alpha \beta^{4}}{45}+\frac{56 e^{\frac{25 t}{7}} \alpha^{2} \beta^{3}}{33}-\frac{931 e^{\frac{22 t}{7}} \alpha^{2} \beta^{3}}{396}-\frac{2240 e^{3 t} \alpha^{2} \beta^{3}}{187}+\frac{51 e^{\frac{18 t}{7}} \alpha^{2} \beta^{3}}{2} \\
& +\frac{2373 e^{2 t} \alpha^{2} \beta^{3}}{220}-\frac{302 e^{\frac{11 t}{7}} \alpha^{2} \beta^{3}}{11}+\frac{196 e^{\frac{10 t}{7}} \alpha^{2} \beta^{3}}{33}-\frac{98 e^{t} \alpha^{2} \beta^{3}}{3} \\
& +\frac{23354 e^{\frac{4 t}{7}} \alpha^{2} \beta^{3}}{765}-\frac{140 e^{\frac{15 t}{7}} \beta^{3}}{11}+14 e^{2 t} \beta^{3}+\frac{35 e^{\frac{12 t}{7}} \beta^{3}}{2}-20 e^{\frac{11 t}{7}} \beta^{3} \\
& +\frac{27 e^{\frac{4 t}{7}} \beta^{3}}{22}+\frac{28 e^{\frac{25 t}{7}} \alpha^{3} \beta^{2}}{33}-\frac{2240 e^{3 t} \alpha^{3} \beta^{2}}{187}+\frac{49 e^{\frac{20 t}{7}} \alpha^{3} \beta^{2}}{12}+\frac{196 e^{\frac{17 t}{7}} \alpha^{3} \beta^{2}}{39} \\
& -\frac{217 e^{2 t} \alpha^{3} \beta^{2}}{165}+\frac{98 e^{\frac{11 t}{7}} \alpha^{3} \beta^{2}}{3}-\frac{49 e^{\frac{10 t}{7}} \alpha^{3} \beta^{2}}{22}-\frac{98 e^{t} \alpha^{3} \beta^{2}}{3}-\frac{49 e^{\frac{6 t}{7}} \alpha^{3} \beta^{2}}{2} \\
& +\frac{132881 e^{\frac{4 t}{7}} \alpha^{3} \beta^{2}}{4420}-\frac{140 e^{\frac{15 t}{7}} \alpha \beta^{2}}{11}+14 e^{2 t} \alpha \beta^{2}+\frac{380 e^{\frac{11 t}{7}} \alpha \beta^{2}}{7} \\
& -\frac{140 e^{\frac{10 t}{7}} \alpha \beta^{2}}{3}-\frac{65 t e^{\frac{4 t}{7}} \alpha \beta^{2}}{7}-\frac{16301 e^{\frac{4 t}{7}} \alpha \beta^{2}}{924}+\frac{35 \alpha \beta^{2}}{4}+\frac{49 e^{\frac{20 t}{7}} \alpha^{4} \beta}{12} \\
& -\frac{49 e^{\frac{16 t}{7}} \alpha^{4} \beta}{9}-\frac{343 e^{2 t} \alpha^{4} \beta}{30}+\frac{245 e^{\frac{10 t}{7}} \alpha^{4} \beta}{9}-\frac{49 e^{\frac{6 t}{7}} \alpha^{4} \beta}{2}+\frac{1813 e^{\frac{4 t}{7}} \alpha^{4} \beta}{180} \\
& +\frac{28 e^{2 t} \alpha^{2} \beta}{5}-\frac{56 e^{\frac{13 t}{7}} \alpha^{2} \beta}{9}-16 e^{\frac{111 t}{7}} \alpha^{2} \beta+\frac{98 e^{\frac{10 t}{7}} \alpha^{2} \beta}{9}+\frac{35 t e^{\frac{4 t}{7}} \alpha^{2} \beta}{3} \\
& -\frac{181 e^{\frac{4 t}{7}} \alpha^{2} \beta}{60}+\frac{35 \alpha^{2} \beta}{4}+\frac{28 e^{2 t} \alpha^{3}}{5}-\frac{56 e^{\frac{13 t}{7}} \alpha^{3}}{9}-\frac{28 e^{\frac{10 t}{7}} \alpha^{3}}{3}+\frac{56 e^{\frac{9 t}{7}} \alpha^{3}}{5} \\
& -\frac{56 e^{\frac{4 t}{7}} \alpha^{3}}{45}-\frac{40 t e^{\frac{4 t}{7}} \alpha}{7}+40 e^{\frac{4 t}{7}} \alpha-40 e^{\frac{3 t}{7}} \alpha \\
& v_{2}(t)=-\frac{490 e^{\frac{22 t}{7}} \alpha \beta^{4}}{99}+\frac{49 e^{\frac{19 t}{7}} \alpha \beta^{4}}{6}-\frac{161 e^{\frac{11 t}{7}} \alpha \beta^{4}}{22}+\frac{49 e^{\frac{8 t}{7}} \alpha \beta^{4}}{8}-\frac{133 e^{\frac{4 t}{7}} \alpha \beta^{4}}{36} \\
& +\frac{147 \alpha \beta^{4}}{88}-\frac{490 e^{\frac{22 t}{7}} \alpha^{2} \beta^{3}}{99}+\frac{784 e^{3 t} \alpha^{2} \beta^{3}}{187}+103 e^{\frac{18 t}{7}} \alpha^{2} \beta^{3}-\frac{1566 e^{\frac{11 t}{7}} \alpha^{2} \beta^{3}}{11} \\
& +\frac{49 e^{\frac{10 t}{7}} \alpha^{2} \beta^{3}}{11}+\frac{49 e^{\frac{8 t}{7}} \alpha^{2} \beta^{3}}{8}-\frac{322 e^{t} \alpha^{2} \beta^{3}}{3}+\frac{127031 e^{\frac{4 t}{7}} \alpha^{2} \beta^{3}}{1224} \\
& +\frac{49 e^{-\frac{4 t}{7}} \alpha^{2} \beta^{3}}{8}+\frac{2373 \alpha^{2} \beta^{3}}{88}+\frac{175 e^{\frac{12 t}{7}} \beta^{3}}{2}-100 e^{\frac{11 t}{7}} \beta^{3}+\frac{145 e^{\frac{4 t}{7}} \beta^{3}}{6} \\
& -\frac{140 e^{\frac{t}{7}} \beta^{3}}{3}+35 \beta^{3}+\frac{784 e^{3 t} \alpha^{3} \beta^{2}}{187}-\frac{3136 e^{\frac{17 t}{7}} \alpha^{3} \beta^{2}}{39}+\frac{5306 e^{\frac{11 t}{7}} \alpha^{3} \beta^{2}}{33} \\
& +\frac{1225 e^{\frac{10 t}{7}} \alpha^{3} \beta^{2}}{33}-\frac{322 e^{t} \alpha^{3} \beta^{2}}{3}+\frac{49 e^{\frac{6 t}{7}} \alpha^{3} \beta^{2}}{3}-\frac{105161 e^{\frac{4 t}{7}} \alpha^{3} \beta^{2}}{2652} \\
& +\frac{49 e^{-\frac{4 t}{7}} \alpha^{3} \beta^{2}}{4}-\frac{217 \alpha^{3} \beta^{2}}{66}+\frac{28 e^{2 t} \alpha \beta^{2}}{55}-81 e^{\frac{11 t}{7}} \alpha \beta^{2}+\frac{280 e^{\frac{10 t}{7}} \alpha \beta^{2}}{3} \\
& +\frac{11 e^{\frac{4 t}{7}} \alpha \beta^{2}}{15}-\frac{21 e^{\frac{3 t}{7}} \alpha \beta^{2}}{11}-\frac{140 e^{\frac{t}{7}} \alpha \beta^{2}}{3}+35 \alpha \beta^{2}+\frac{98 e^{\frac{16 t}{7}} \alpha^{4} \beta}{9} \\
& -\frac{343 e^{\frac{10 t}{7}} \alpha^{4} \beta}{9}+\frac{49 e^{\frac{6 t}{7}} \alpha^{4} \beta}{3}+\frac{2401 e^{\frac{4 t}{7}} \alpha^{4} \beta}{72}+\frac{49 e^{-\frac{4 t}{7}} \alpha^{4} \beta}{8}-\frac{343 \alpha^{4} \beta}{12} \\
& +\frac{28 e^{2 t} \alpha^{2} \beta}{55}-80 e^{\frac{11 t}{7}} \alpha^{2} \beta+92 e^{\frac{10 t}{7}} \alpha^{2} \beta-\frac{62 e^{\frac{4 t}{7}} \alpha^{2} \beta}{5}-\frac{32 e^{\frac{3 t}{7}} \alpha^{2} \beta}{11} \\
& -\frac{56 e^{-\frac{t}{7}} \alpha^{2} \beta}{5}+14 \alpha^{2} \beta-\frac{40 t e^{\frac{4 t}{7}} \beta}{7}+40 e^{\frac{4 t}{7}} \beta-40 e^{\frac{3 t}{7}} \beta+\frac{56 e^{\frac{10 t}{7}} \alpha^{3}}{3}
\end{aligned}
$$




$$
-\frac{112 e^{\frac{9 t}{7}} \alpha^{3}}{5}+\frac{14 e^{\frac{4 t}{7}} \alpha^{3}}{15}-\frac{56 e^{-\frac{t}{7}} \alpha^{3}}{5}+14 \alpha^{3}
$$

The approximate solution of (16) by HPM is given by:

$$
\begin{aligned}
u(t) & \simeq \lim _{p \rightarrow 1}\left[u_{0}(t)+p u_{1}(t)+p^{2} u_{2}(t)\right] \\
& =u_{0}(t)+u_{1}(t)+u_{2}(t)
\end{aligned}
$$

and

$$
\begin{aligned}
v(t) & \simeq \lim _{p \rightarrow 1}\left[v_{0}(t)+v u_{1}(t)+p^{2} v_{2}(t)\right] \\
& =v_{0}(t)+v_{1}(t)+v_{2}(t)
\end{aligned}
$$

\section{Numerical Results and Discussion}

For the example 1, we get its exact solution with both methods (HPM and SBA method). For example 2, in oder to verify the efficiency of the proposed method in comparison with the exact solution, we calculate the values of these solutions for different values of $t$.

The differences between HPM solution and exact solution are shown in tables (1),(2) and the figures (1), (2), and (3). We can see a good agreement between the results of HPM and the SBA method, which confirms the validity of HPM.

Table 1. Example 2: approximate solution of $u$ by SBA and HPM

\begin{tabular}{cccc}
\hline$t$ & $u_{s b a}$ & $u_{h p m}$ & $\left|u_{s b a}-u_{h p m}\right|$ \\
\hline 0 & $0.0060 \times 10^{9}$ & $0.0060 \times 10^{9}$ & 0 \\
\hline 0.5 & $0.0349 \times 10^{9}$ & $0.0349 \times 10^{9}$ & 0 \\
\hline 1.0 & $0.0727 \times 10^{9}$ & $0.0727 \times 10^{9}$ & 0 \\
\hline 1.5 & $0.1291 \times 10^{9}$ & $0.1291 \times 10^{9}$ & 0 \\
\hline 2.0 & $0.2184 \times 10^{9}$ & $0.2183 \times 10^{9}$ & $0.0010 \times 10^{8}$ \\
\hline 2.5 & $0.3635 \times 10^{9}$ & $0.3629 \times 10^{9}$ & $0.0060 \times 10^{8}$ \\
\hline 3.0 & $0.6014 \times 10^{9}$ & $0.5981 \times 10^{9}$ & $0.0330 \times 10^{8}$ \\
\hline 3.5 & $0.9927 \times 10^{9}$ & $0.9791 \times 10^{9}$ & $0.1360 \times 10^{8}$ \\
\hline 4.0 & $1.6375 \times 10^{9}$ & $1.5902 \times 10^{9}$ & $0.4730 \times 10^{8}$ \\
\hline 4.5 & $2.7002 \times 10^{9}$ & $2.5573 \times 10^{9}$ & $1.4290 \times 10^{8}$ \\
\hline 5.0 & $4.4522 \times 10^{9}$ & $4.0639 \times 10^{9}$ & $3.8830 \times 10^{8}$ \\
\hline
\end{tabular}

Table 2. Example 2: approximate solution of $v$ by SBA and HPM

\begin{tabular}{cccc}
\hline$t$ & $v_{s b a}$ & $v_{h p m}$ & $\left|v_{s b a}-v_{h p m}\right|$ \\
\hline 0 & $0.0360 \times 10^{9}$ & $0.0360 \times 10^{9}$ & 0 \\
\hline 0.5 & $0.0343 \times 10^{9}$ & $0.0343 \times 10^{9}$ & 0 \\
\hline 1.0 & $0.0414 \times 10^{9}$ & $0.0414 \times 10^{9}$ & 0 \\
\hline 1.5 & $0.0591 \times 10^{9}$ & $0.0591 \times 10^{9}$ & 0 \\
\hline 2.0 & $0.0919 \times 10^{9}$ & $0.0919 \times 10^{9}$ & 0 \\
\hline 2.5 & $0.1482 \times 10^{9}$ & $0.1482 \times 10^{9}$ & 0 \\
\hline 3.0 & $0.2422 \times 10^{9}$ & $0.2423 \times 10^{9}$ & $0.0100 \times 10^{7}$ \\
\hline 3.5 & $0.3981 \times 10^{9}$ & $0.3980 \times 10^{9}$ & $0.0100 \times 10^{7}$ \\
\hline 4.0 & $0.6556 \times 10^{9}$ & $0.6539 \times 10^{9}$ & $0.1700 \times 10^{7}$ \\
\hline 4.5 & $1.0805 \times 10^{9}$ & $1.0712 \times 10^{9}$ & $0.9300 \times 10^{7}$ \\
\hline 5.0 & $1.7811 \times 10^{9}$ & $.7450 \times 10^{9}$ & $3.6100 \times 10^{7}$ \\
\hline
\end{tabular}




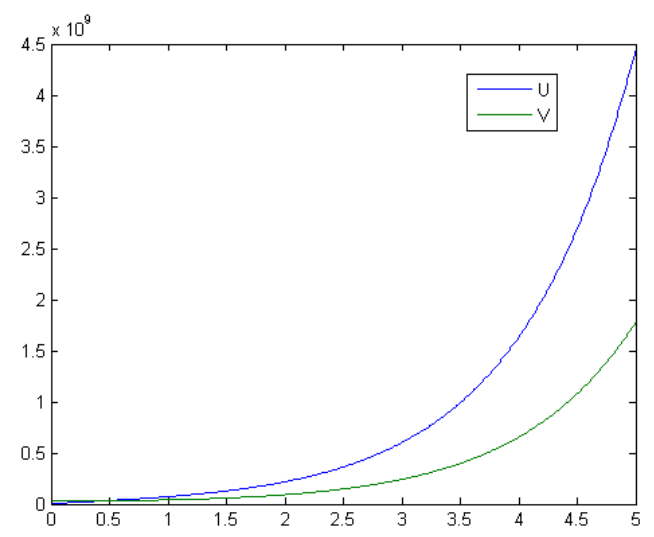

(a) SBA solution

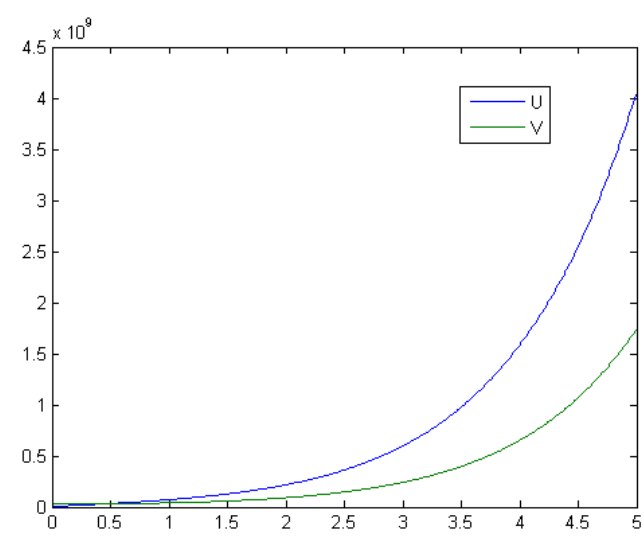

(b) HPM solution

Figure 1. Example2 : Comparison of HPM and SBA method solution

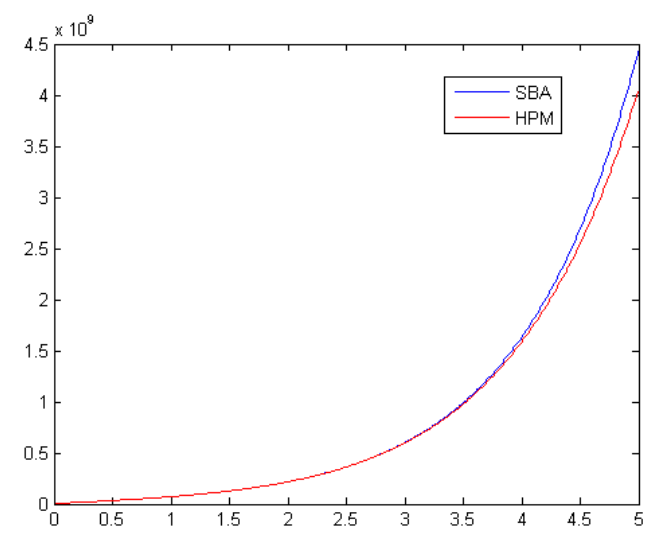

(a) $u$

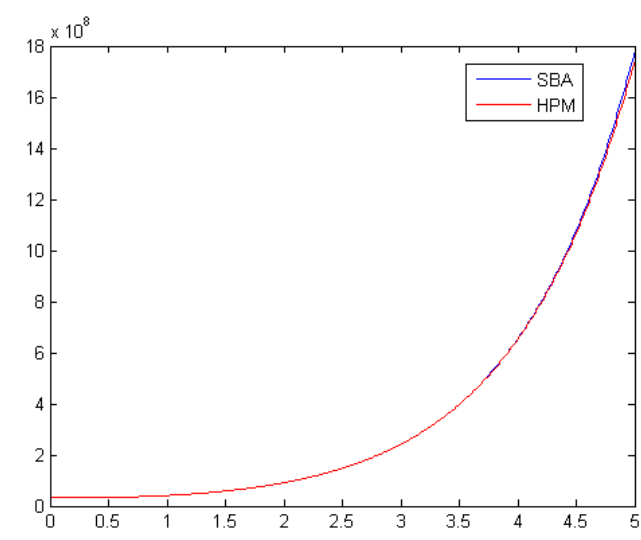

(b) $v$

Figure 2. Example2 : Comparison of HPM and SBA method solution

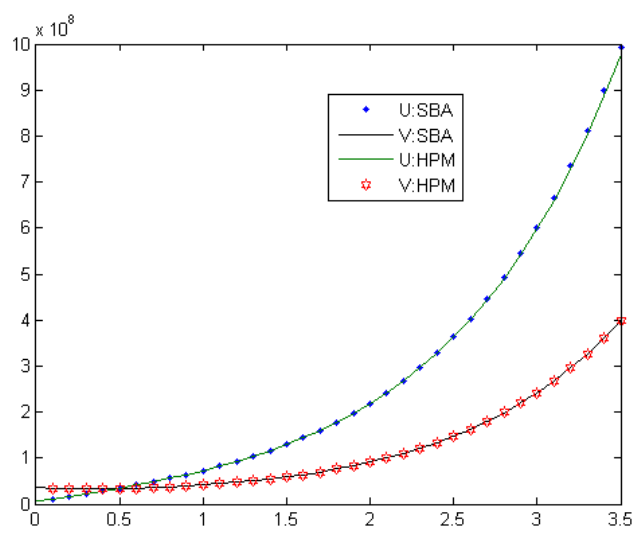

(a) $u$

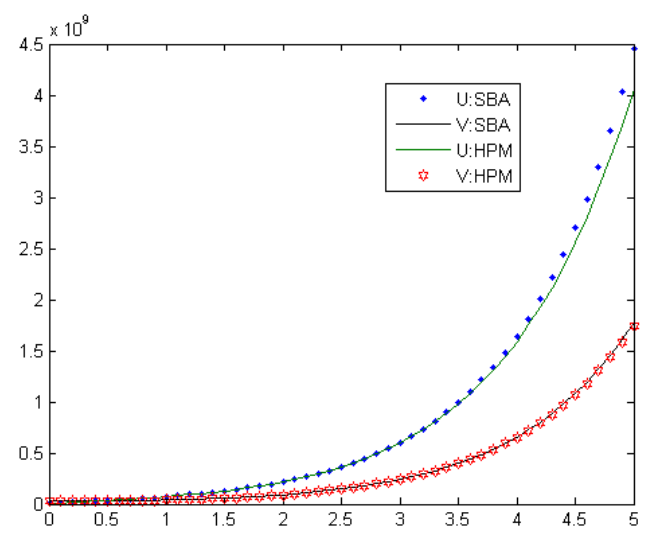

(b) $v$

Figure 3. Example2 : Comparison of HPM and SBA method solution 


\section{Conclusion}

In this paper, the homotopy perturbation method was used for finding exact or approximate solutions of Lotka-Volterra models. Through the examples studied, we have shown that we obtain practically the same solutions with HPM and the SBA method.

\section{References}

Abbo, B. (2007). Nouvel algorithme numérique de résolution des équations différentielles ordinaires (EDO) et des équations aux drivées partielles non linéaires Thése de Doctorat unique. Université de Ouagadougou.

BAGAYOGO, M. (2019). Recherche et comparaison d'une solution approchée de certaines équations de la physique mathématique par les méthodes de l'homotopie des perturbations (HPM), des perturbations réguliéres (RPM) et d'Adomian (ADM). Thèse de doctorat de l'Université Joseph Ki-Zerbo.

Bagayogo, M., Pare, Y., \& Minoungou, Y. (2018). An Approached Solution of Wave Equation with Cubic Damping by Homotopy Perturbation Method (HPM), Regular Pertubation Method (RPM) and Adomian Decomposition Method (ADM). Journal of Mathematics Research, 10(2), 166-181. https://doi.org/10.5539/jmr.v10n2p166

BAGAYOGO, M., MINOUNGOU, Y., PARE, Y., \& SOME, B. (2019). An analytical solution of perturbed Fisher's equation using Homotopy Perturbation Method (HPM), Regular Perturbation Method (RPM) and Adomian Decomposition Method (ADM), Australian Journal of Mathematical Analysis and Applications, 16(1), 1-17.

BISSANGA, G. (2005). Application of the Adomian Decomposition Method to solving the van der Pol equation.Comparison with the Regular Perturbation Method. Proceedings of the Fourth International Workshop on Contemporary Problems in Mathematical Physics, Cotonou Benin. World Scientific Publishing Co. Pte. Ltd.

Chowdhury, M. S. H., Hashim, I., \& Ismail, A. F. (2010, June). Analytical Treatment of System of Linear and Nonlinear PDEs by Homotopy-Perturbation Method. In Proceedings of the World congress on Engineering (Vol. 3).

Chowdhury, M. S. H., Razali, N. I., Asrar, W., \& Rahman, M. M. (2015, August). The multistage homotopy perturbation method for solving chaotic and hyperchaotic 1 system. In Abstract and Applied Analysis (Vol. 2015). Hindawi.

Cushing, J. M., \& Saleem, M. (1982). A Predator Prey Model with Age Structure. J. Math. Biol., 14, 231-250.

KADEM, A., \& BALEANU, D. (2011). Homotopy perturbation method for the coupled fractional Lotka-Volterra equations. Rom. Journ. Phys., 56(3-4), 332-338.

MINOUNGOU, Y. (2019). Contribution des méthodes SBA, Laplace-SBA la résolution des modéles de convectionDiffusion-Réaction. Thèse de doctorat de l'Université Joseph Ki-Zerbo.

PARE, Y. (2010). Résolution de quelques équations fonctionnelles par la numérique SBA (Somé Blaise-Abbo). Thése de Doctorat unique. Université de Ouagadougou.

Nourazar, S. S., Sooril, M., \& Golshan, A. N. (2016). On the Homotopy Perturbation Method for the Exact Solution of Fitzhugh Nagumo Equation. International Journal of Mathematics and Computation, 27(1), 32-43.

YARO, R. (2016) Contribution la résolution de quelques équations fonctionnelles et modéles mathématiques de la dynamique des populations par les méthodes d'Adomian, SBA et des perturbations. Thèse de doctorat de l'Université Ouaga Pr Joseph Ki-Zerbo.

\section{Copyrights}

Copyright for this article is retained by the author(s), with first publication rights granted to the journal.

This is an open-access article distributed under the terms and conditions of the Creative Commons Attribution license (http://creativecommons.org/licenses/by/4.0/). 